\title{
Global Antifungal Profile Optimization of Chlorophenyl Derivatives against Botrytis cinerea and Colletotrichum gloeosporioides
}

\author{
Liane Saiz-Urra, ${ }^{*}$ Antonio J. Bustillo Pérez, ${ }^{\dagger}$ Maykel Cruz-Monteagudo, ${ }^{\ddagger}, \$$ \\ Cristina Pinedo-Rivilla, ${ }^{\dagger}$ Josefina Aleu $^{\dagger}$ Rosario Hernández-Galán, $^{\dagger}$ and \\ ISIDRo G. COLlado*, ${ }^{\dagger}$
}

†Departamento de Química Orgánica, Facultad de Ciencias, Universidad de Cádiz, Apartado 40, 11510 Puerto Real, Cádiz, Spain, ${ }^{\star}$ Chemical Bioactive Center and Applied Chemistry Research Center, Faculty of Chemistry and Pharmacy, Central University of Las Villas, Santa Clara, Villa Clara, C.P. 54830, Cuba, and ${ }^{\S}$ Department of Organic Chemistry, Faculty of Pharmacy, University of Porto, 4150-047 Porto, Portugal

\begin{abstract}
Twenty-two aromatic derivatives bearing a chlorine atom and a different chain in the para or meta position were prepared and evaluated for their in vitro antifungal activity against the phytopathogenic fungi Botrytis cinerea and Colletotrichum gloeosporioides. The results showed that maximum inhibition of the growth of these fungi was exhibited for enantiomers $S$ and $R$ of 1 -( $4^{\prime}$-chlorophenyl)2-phenylethanol (3 and $\mathbf{4})$. Furthermore, their antifungal activity showed a clear structure-activity relationship (SAR) trend confirming the importance of the benzyl hydroxyl group in the inhibitory mechanism of the compounds studied. Additionally, a multiobjective optimization study of the global antifungal profile of chlorophenyl derivatives was conducted in order to establish a rational strategy for the filtering of new fungicide candidates from combinatorial libraries. The MOOPDESIRE methodology was used for this purpose providing reliable ranking models that can be used later.
\end{abstract}

KEYWORDS: Chlorophenyl derivatives; crop-protection agents; Botrytis cinerea; Colletotrichum gloeosporioides; antifungal activity; MOOP-DESIRE methodology; 3D molecular descriptors

\section{INTRODUCTION}

While increased resistance to commercially available agrochemicals has led to the need for new fungicides, the desire for safer and more effective agrochemicals with reduced environmental toxicity also remains a high priority. Essential to fulfilling both of these needs is the identification of new lead candidates that possess high levels of desirable biological activities, reduced levels of unwanted toxicities, new structural types, and perhaps different modes of action in order to avoid the problem of cross-resistance, which is so prevalent among currently used agrochemicals (1). In this context, natural product-based fungicides offer advantages in that they have unique modes of action as well as low mammalian toxicity. An additional benefit is their ability to decompose rapidly, thereby reducing environmental risk. Since phytoalexins are known to exhibit significant antifungal activity (2), we have undertaken a screening of analogous compounds (3) in order to find substrates with similar antifungal properties.

Our investigations focused on the metabolism of diverse compounds, which are analogous to the phytoalexins produced by the agriculturally important pathogens Botrytis cinerea and Colletotrichum gloeosporioides. These two species are highly destructive

*Corresponding author. Tel: 34956 016368. Fax: 34956016193. E-mail: isidro.gonzalez@uca.es. plant-pathogenic fungi, responsible for diseases such as gray mold in grapes and anthracnose affecting strawberry fruits and plants $(4,5)$. On the basis of previous results indicating that the presence of a hydroxyl group was determined to be fundamental for the expression of the antifungal activity of the compounds studied (6-9), we decided to study compounds containing a secondary hydroxyl group or a ketone in their structure.

A series of chlorophenyl derivatives were prepared and several of these incubated with $B$. cinerea $(7,10)$ and C. gloeosporioides (10). The existence of a microbial detoxification pathway for these fungistatic agents suggests that they might not persist in the environment for a prolonged period $(7,10)$.

In order to rationalize results and to suggest further optimization of chlorophenyl derivative activity, a MOOP-DESIREbased optimization of the global antifungal profile was done taking both microorganisms into account. In this article, we report the application of this methodology to this field for the first time with the aim of rationalizing the procedure to obtain new chemicals with a broad antifungal spectrum.

\section{MATERIALS AND METHODS}

General Experimental Procedures. Optical rotations were determined with a Perkin-Elmer 241 polarimeter. IR spectra were recorded on a Mattson Genesis spectrophotometer, series FTIR. ${ }^{1} \mathrm{H}$ and ${ }^{13} \mathrm{C}$ 
NMR measurements were obtained on a Varian Inova 400 NMR and $600 \mathrm{MHz}$ spectrometers with $\mathrm{SiMe}_{4}$ as the internal reference. Mass spectra were recorded on a GC-MS Thermoquest spectrometer (Voyager model) and a VG Autospec-Q spectrometer. HPLC was performed with a Hitachi/Merck L-6270 apparatus equipped with a UV-vis detector ( $\mathrm{L} 4250$ ) and a differential refractometer detector (RI-71). TLC was performed on a Merck Kiesegel $60 \mathrm{~F}_{254}, 0.2 \mathrm{~mm}$ in thickness. Silica gel (Merck) was used for column chromatography. Purification by means of HPLC was accomplished using a silica gel column (Hibar 60, $7 \mathrm{~m}, 1 \mathrm{~cm}$ wide, $25 \mathrm{~cm}$ long). Chemicals were products of Fluka or Aldrich. All solvents used were freshly distilled. Enantiomeric excesses were determined by means of HPLC analyses on a chiral column (Chiralcel OD, Daicel, Japan).

Obtaining Chlorophenyl Derivatives. Ketones 1, 9, 13, and 18 are commercial products. Racemic alcohols 2, 10, 14, and 19 were prepared from the corresponding ketones according to a previously described procedure (3). Enantiopure compounds 15-17 and 20-22 were obtained through biological methods (3). The rest of the products, $\mathbf{3}-\mathbf{8}$, $\mathbf{1 1}$, and $\mathbf{1 2}$, were prepared by chemical methods (10).

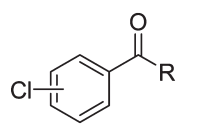

1: 4' $\mathrm{Cl} ; \mathrm{R}=\mathrm{Bn}$ 9: $4^{\prime} \mathrm{Cl} ; \mathrm{R}=\mathrm{Z}$ 13: 4' $\mathrm{Cl}$; $\mathrm{R}=\mathrm{E}$ 18: $3^{\prime} \mathrm{Cl} ; \mathrm{R}=\mathrm{Et}$



2: $4^{\prime} \mathrm{Cl} ; \mathrm{R}=\mathrm{OH} ; \mathrm{R}^{\prime}=\mathrm{Bn}$

3: $4^{\prime} \mathrm{Cl} ; \mathrm{R}=\beta \mathrm{OH} ; \mathrm{R}^{\prime}=\mathrm{Bn}$ 4: 4' $\mathrm{Cl} ; \mathrm{R}=\alpha \mathrm{OH} ; \mathrm{R}^{\prime}=\mathrm{Bn}$ 10: 4' $\mathrm{Cl}$; $\mathrm{R}=\mathrm{OH} ; \mathrm{R}^{\prime}=$ 11: $4^{\prime} \mathrm{Cl} ; \mathrm{R}=\alpha \mathrm{OH} ; \mathrm{R}^{\prime}=$ 12: 4' $\mathrm{Cl}$; $\mathrm{R}=\beta \mathrm{OH}$; R'= 14: 4 ' $\mathrm{Cl} ; \mathrm{R}=\mathrm{OH} ; \mathrm{R}^{\prime}=\mathrm{Et}$ 15: 4' $\mathrm{Cl}$; $R=\beta \mathrm{OH}$; $\mathrm{R}^{\prime}=\mathrm{Et}$ 16: $4^{\prime} \mathrm{Cl} ; \mathrm{R}=\alpha \mathrm{OH} ; \mathrm{R}^{\prime}=\mathrm{Et}$ 17: 4' $\mathrm{Cl} ; \mathrm{R}=\alpha \mathrm{OAc} ; \mathrm{R}^{\prime}=\mathrm{Et}$ 19: $3^{\prime} \mathrm{Cl} ; \mathrm{R}=\mathrm{OH} ; \mathrm{R}^{\prime}=\mathrm{Et}$ 20: $3^{\prime} \mathrm{Cl} ; \mathrm{R}=\beta \mathrm{OH} ; \mathrm{R}^{\prime}=\mathrm{Et}$ 21: 3 ' Cl; $\mathrm{R}=\alpha \mathrm{OH} ; \mathrm{R}^{\prime}=\mathrm{Et}$ 22: 3' Cl; R= $\alpha$ OAc; $R^{\prime}=E t$

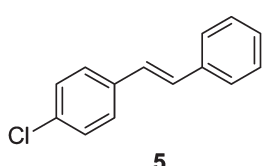

5

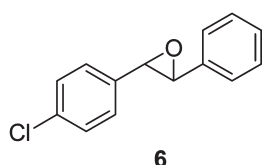

6

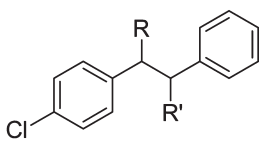

7: $\mathrm{R}=\mathrm{R}=\beta \mathrm{OH}$ 8: $\mathrm{R}=\mathrm{R}^{\prime}=\alpha \mathrm{OH}$
Microorganism and Antifungal Assays. B. cinerea and $C$. gloeosporioides cultures used in this work, B. cinerea 2100 and $C$. gloeosporioides 20122-CECT, respectively, were obtained from the Centro Español de Cultivos Tipo (CECT), Facultad de Biología, Universidad de Valencia, Spain, where cultures of these strains are on deposit. Bioassays were performed by measuring radial growth inhibition on agar medium in a Petri dish at $25{ }^{\circ} \mathrm{C}$. Test compounds were dissolved in $\mathrm{EtOH}$ to give a final compound concentration in the culture medium of 10 to $150 \mathrm{mg} \mathrm{L}^{-1}$. Solutions of the test compounds were added to a glucose-malt-peptone-agar medium (61 $\mathrm{g}$ of glucose-malt-peptone-agar per L, pH 6.5-7.0). The final EtOH concentration was identical in both the control and treated cultures. The medium was poured into 6- or 9-cm diameter sterile plastic Petri dishes, and a 6-mm diameter mycelial disk of the fungus cut from an actively growing culture was placed in the center of the agar plate. Inhibition of radial growth was measured for 10 days. Results are the means of at least three independent experiments conducted in triplicate. The commercial fungicide Euparen $\left(50,100\right.$, and $150 \mathrm{mg} \mathrm{L}^{-1}$ ) was used as a standard for comparison in this test.

MOOP-DESIRE-Based Optimization of the Global Antifungal

Profile. The conventional methods of trial and error to obtain new antifungal compounds are very expensive and take a long time to be carried out. Therefore, theoretical computational methods such as QSAR studies have become an alternative capable of saving time and resources in the antifungal development process allowing an efficient and rapid progress $(11,12)$.

The MOOP-DESIRE methodology was employed here to simultaneously optimize and rank a set of candidates according to their global antifungal profile against $B$. cinerea and $C$. gloeosporioides. The details of this procedure can be found in refs (13 and 14).
This approach consists of three main steps including several tasks:

I. development of predictive models (PMs) as good as possible in terms of their statistical significance, robustness, and predictive ability for each response;

II. conversion of the desirability $\left(d_{i}\right)$ of every compound attending to each biological response on the basis of the PMs developed on the first step into the global desirability $\left(D_{i}\right)$; then, this is used to attain the levels of descriptors for the optimal candidate.

III. These results are then used as a template for a ranking algorithm based on quantitative parameters estimated from the description of the cases in order to rank candidates with unknown antifungal profiles.

The main advantage of this methodology is that it is possible to rank new (unknown fungicide) candidates based solely on structural information. It will hence be possible to filter and identify the most promising candidates, which naturally will be placed at the top of the list (the candidates with the lowest $\Delta_{i}$ values and consequently the ones most similar to the optimal candidate determined by the desirability-based MOOP process $(13,14))$, and to discard the candidates at the bottom of the list. It is important to emphasize that as in any other computational approach for predicting biological activities, it is necessary to verify the reliability of the models by means of validations techniques $(13,14)$.

In this work, two desirability functions $\left(d_{i}\right)$, one for each response, were fitted. Specifically, antifungal activity against $B$. cinerea and $C$. gloeosporioides ought to be maximized. For the estimation of the two $d_{i}$ values, the lower value $L_{i}$ was set to $20 \%$ and $25 \%$. The upper value $U_{i}$, made equal to the target value $T_{i}$, was set to $70 \%$ and $90 \%$ for each response. Furthermore, the spline method $(15,16)$ was used to fit the desirability function, and the current level of each independent variable was set equal to its optimum value. The $s$ parameter was fixed at 1.00 by assuming that the desirability functions increase linearly toward $T_{i}$ for both responses.

Once the kind of function for each response was defined, the global desirability $D$ of each $i$-th candidate was evaluated to achieve the overall assessment of the desirability of the combined response levels. The optimization of such a function was carried out via the general use optimization function option of the STATISTICA (17) general regression module. Then, weights were obtained by means of a nonlinear curve-fitting using the large-scale optimization algorithm $(18,19)$ implemented in the 1sqcurvefit function of the MATLAB program, version 7.2(20). Details on the validation of the ranking algorithm employed as well as the definition and determination of $\Psi$ can be found in the references $(13,14)$.

Computational Methods. Because of the large number of stereoisomers in the data set, we decided to use 3D molecular descriptors to account for their antifungal activity based on the different configurations. The structures corresponding to the racemic compounds $2,10,14$, and 19 were excluded from the data set to avoid possible noise in the fitness of the model since the antifungal activity of the separated enantiomers was available. The reasonable starting geometries were obtained by resorting to the MM2 molecular mechanics force field $(21,22)$. Molecular structures were then fully optimized with the PM3 semiempirical Hamiltonian (23) implemented in the MOPAC 6.0 program (24).

The optimized structures were then uploaded to the DRAGON software package (25) to compute a total of 721 3D molecular descriptors. As part of the necessary variable reduction, descriptors with constant or nearly constant values and those which were highly pair-correlated $(|R|>$ 0.95 ) were excluded, with the final pool consisting of 649 descriptors.

The variable selection approach used in this work to establish the QSAR models was the genetic algorithm (GA) (26-29) by means of the BuildQSAR software package $(30,31)$. Table 1 depicts the DRAGON molecular descriptors of the chlorophenyl derivatives selected in this study to model antifungal properties against B. cinerea and $C$. gloeosporioides.

As for the modeling technique, we opted for a regression-based approach; in this case, the regression coefficients and statistical parameters were obtained by multiple linear regression (MLR) analysis using the STATISTICA software package (17). Because of the need for more potent and less persistent new antifungal drugs, we considered their activity reported at $50 \mu \mathrm{g} / \mathrm{mL}$. Also, the dependent variable was evaluated as the $\log$ of the inhibition percentage plus one in order to establish a linear relationship between the activity and the structures under study and avoid 
Table 1. Descriptors Involved in the Models Reported in This Study

\begin{tabular}{ll}
\hline symbol & \multicolumn{1}{c}{ definition } \\
\hline Mor27v & 3D-MoRSE-signal 27/weighted by atomic van der Waals volumes \\
H5e & H autocorrelation of lag 5/weighted by atomic Sanderson \\
& electronegativities \\
R4e & R autocorrelation of lag 4/weighted by atomic Sanderson \\
& electronegativities \\
SP18 & shape profile no. 18 \\
QYYe & $\begin{array}{l}\text { Qyy COMMA2 value/weighted by atomic Sanderson } \\
\text { electronegativities }\end{array}$ \\
R7e & R autocorrelation of lag 7/weighted by atomic Sanderson \\
& electronegativities \\
\hline
\end{tabular}

the indetermination of the logarithmic function when the percentage values are equal to zero.

The goodness of fit for each predictive model (PM) was assessed by examining the determination coefficient $\left(R^{2}\right)$, the adjusted determination coefficient $\left(A d j . R^{2}\right)$, the standard deviation ( $(s)$, Fisher's statistics $(F)$, and the ratio between the number of compounds $(N)$ and the number of adjustable parameters $(\dot{p})$ in the model known as the $\rho$ statistics. The stability and predictive ability of the models were addressed by means of internal cross-validation (CV), specifically using the leave-one-out (LOO) $(32,33)$ and bootstrapping techniques $(34)$. The overall desirability determination coefficient for training $\left(R_{D}^{2}\right)$ and leave-one-out cross validation $\left(Q_{D}^{2}\right)$ were used to test uncertainty in predicting the overall desirability function and the reliability of the simultaneous optimization of the $k$ responses over the independent variables domain, respectively (14).

\section{RESULTS AND DISCUSSION}

Twenty-two aromatic derivatives bearing a chlorine atom and a different chain in the para or meta position were prepared and evaluated for their in vitro antifungal activity against the phytopathogenic fungi B. cinerea and C. gloeosporioides.

We had previously shown that $( \pm)-1-\left(4^{\prime}\right.$-chlorophenyl)-2-phenylethanol (2) and $(R)-(+)-1-\left(4^{\prime}\right.$-chlorophenyl)propan-1-ol (16), compounds analogous to several phytoalexins, are active fungistatic agents against $B$. cinerea and $C$. gloeosporioides $(3,35)$. Detoxification by these fungi was studied and suggests that they probably would not persist in the environment for long periods post-application $(7,10)$.

On the basis of previous results, from which the presence of a hydroxyl group was determined to be fundamental for the expression of the antifungal activity of the compounds studied (6-9), we chose to study compounds that contain a secondary hydroxyl group or a ketone in their structure. Thus, compounds $\mathbf{1 - 2 2}$ were obtained as previously reported $(3,10)$.

The antifungal properties of chlorophenyl derivatives were determined against the growth of $B$. cinerea and $C$. gloeosporioides using the poisoned food technique (36). Several levels of inhibition were observed. The commercial fungicide Euparen (50, 100 , and $150 \mathrm{mg} \mathrm{L}^{-1}$ ) was used as a standard for comparison in this test, showing $100 \%$ inhibition for both fungi at all of the concentrations.

According to Tables $\mathbf{2}$ and $\mathbf{3}$, a generalized minor activity of chlorophenyl derivatives was observed against $C$. gloeosporioides as compared to $B$. cinerea. For both fungi, poor results were obtained for the epoxy and double bond derivatives at C-1 and C2, compounds $\mathbf{5}$ and $\mathbf{6}$, respectively, and the acetyl derivatives $\mathbf{1 7}$ and 22, indicating that the hydroxyl group plays an important role in the inhibitory mechanism.

Also, a decrease in the activity against both fungi was observed when a second hydroxyl group was added to the structure, compounds $\mathbf{7}$ and $\mathbf{8}$, exhibiting reduced activity with respect to the monoalcohols $\mathbf{2}, \mathbf{3}$, and $\mathbf{4}$.

The results of the antifungal activity screening test indicated that in general the monohydroxylated derivatives, 2, 3, 4, 10, 19,
Table 2. Results of Antifungal Bioassays of Compounds 1-22 against Botrytis cinerea (after 5 Days)

\begin{tabular}{lrrr}
\hline & \multicolumn{3}{c}{$\%$ inhibition $\pm \mathrm{SD}^{a}$} \\
\cline { 2 - 4 } comp. & $150 \mu \mathrm{g} / \mathrm{mL}$ & $100 \mu \mathrm{g} / \mathrm{mL}$ & $50 \mu \mathrm{g} / \mathrm{mL}$ \\
\hline $\mathbf{1}$ & $38 \pm 1$ & $33 \pm 1$ & $31 \pm 3$ \\
$\mathbf{2}$ & $100 \pm 0$ & $100 \pm 0$ & $95 \pm 1$ \\
$\mathbf{3}$ & $100 \pm 0$ & $100 \pm 0$ & $91 \pm 2$ \\
$\mathbf{4}$ & $100 \pm 0$ & $100 \pm 0$ & $99 \pm 1$ \\
$\mathbf{5}^{b}$ & $19 \pm 3$ & $17 \pm 2$ & $13 \pm 0$ \\
$\mathbf{6}$ & $28 \pm 2$ & $26 \pm 2$ & $24 \pm 1$ \\
$\mathbf{7}$ & $62 \pm 1$ & $59 \pm 1$ & $32 \pm 1$ \\
$\mathbf{8}$ & $68 \pm 1$ & $63 \pm 2$ & $33 \pm 1$ \\
$\mathbf{9}$ & $100 \pm 0$ & $99 \pm 0$ & $88 \pm 1$ \\
10 & $100 \pm 0$ & $100 \pm 0$ & $74 \pm 3$ \\
11 & $100 \pm 0$ & $100 \pm 0$ & $77 \pm 1$ \\
$\mathbf{1 2}$ & $97 \pm 3$ & $80 \pm 2$ & $63 \pm 1$ \\
$\mathbf{1 3}$ & $91 \pm 0$ & $51 \pm 1$ & $28 \pm 2$ \\
14 & $95 \pm 3$ & $77 \pm 2$ & $61 \pm 1$ \\
$\mathbf{1 5}$ & $92 \pm 1$ & $73 \pm 1$ & $62 \pm 1$ \\
16 & $98 \pm 2$ & $82 \pm 1$ & $74 \pm 1$ \\
$\mathbf{1 7}$ & $44 \pm 1$ & $40 \pm 3$ & $12 \pm 1$ \\
18 & $91 \pm 1$ & $49 \pm 2$ & $26 \pm 1$ \\
19 & $83 \pm 2$ & $77 \pm 1$ & $67 \pm 1$ \\
$\mathbf{2 0}$ & $82 \pm 1$ & $71 \pm 1$ & $64 \pm 2$ \\
$\mathbf{2 1}$ & $86 \pm 1$ & $80 \pm 1$ & $72 \pm 2$ \\
$\mathbf{2 2}$ & $30 \pm 1$ & $29 \pm 2$ & $22 \pm 3$ \\
\hline
\end{tabular}

$a \%$ Inhibition $=100-[$ (growth on treated/growth on control $) \times 100] \pm S D$; the results are the means of at least three independent experiments conducted in triplicate. ${ }^{b} \%$ Inhibition after 4 days.

Table 3. Results of Antifungal Bioassays of Compounds 1-22 against Colletotrichum gloeosporioides (after 5 Days)

\begin{tabular}{lccc}
\hline & \multicolumn{3}{c}{$\%$ inhibition $\pm \mathrm{SD}^{a}$} \\
\cline { 2 - 4 } comp. & $150 \mu \mathrm{g} / \mathrm{mL}$ & $100 \mu \mathrm{g} / \mathrm{mL}$ & $50 \mu \mathrm{g} / \mathrm{mL}$ \\
\hline $1^{b}$ & $17 \pm 3$ & $13 \pm 1$ & $9 \pm 1$ \\
2 & $94 \pm 1$ & $87 \pm 2$ & $63 \pm 2$ \\
3 & $96 \pm 2$ & $85 \pm 2$ & $66 \pm 1$ \\
4 & $100 \pm 0$ & $94 \pm 2$ & $73 \pm 1$ \\
$\mathbf{5}$ & 0 & 0 & 0 \\
6 & 0 & 0 & 0 \\
7 & $51 \pm 2$ & $42 \pm 1$ & $22 \pm 2$ \\
8 & $62 \pm 3$ & $52 \pm 2$ & $27 \pm 1$ \\
9 & $86 \pm 1$ & $63 \pm 3$ & $21 \pm 1$ \\
10 & $80 \pm 2$ & $53 \pm 4$ & $31 \pm 1$ \\
11 & $52 \pm 1$ & $40 \pm 2$ & $25 \pm 2$ \\
$12^{b}$ & $27 \pm 3$ & $23 \pm 2$ & $20 \pm 1$ \\
13 & $36 \pm 1$ & $29 \pm 2$ & $28 \pm 1$ \\
14 & $51 \pm 2$ & $41 \pm 1$ & $28 \pm 1$ \\
15 & $33 \pm 3$ & $29 \pm 2$ & $28 \pm 1$ \\
16 & $59 \pm 2$ & $39 \pm 1$ & $28 \pm 1$ \\
17 & 0 & 0 & 0 \\
18 & $42 \pm 2$ & $28 \pm 1$ & $22 \pm 2$ \\
19 & $62 \pm 2$ & $60 \pm 0$ & $31 \pm 1$ \\
20 & $49 \pm 2$ & $37 \pm 1$ & $32 \pm 2$ \\
21 & $65 \pm 1$ & $60 \pm 3$ & $34 \pm 1$ \\
$\mathbf{2 2}$ & 0 & 0 & 0 \\
\hline
\end{tabular}

${ }^{a} \%$ Inhibition $=100-[($ growth on treated/growth on control $) \times 100] \pm S D$; the results are the means of at least three independent experiments conducted in triplicate. ${ }^{b} \%$ Inhibition after 4 days.

and 21, displayed the best antifungal activity (see Tables $\mathbf{2}$ and $\mathbf{3}$ ) against the growth of both $B$. cinerea and C. gloeosporioides.

In general, the relative position of the chlorine atom in the aromatic ring appears to be important, specially for $B$. cinerea because para-sustituted compounds are more active than the 
Table 4. Regression Coefficients and Statistical Parameters for the MLR Models

Antifungal Activity against $B$. cinerea: MLR Model

\begin{tabular}{|c|c|c|c|c|c|c|c|}
\hline \multicolumn{8}{|c|}{$\log \left(1+\% I_{50 \mathrm{ppm}}\right)=1.020( \pm 0.057)-0.886( \pm 0.161) \cdot$ Mor27v $-1.900( \pm 0.113) \cdot H 5 e+0.830( \pm 0.044) \cdot R 4 e$} \\
\hline$N$ & $R^{2}$ & $R^{2}$ Adj. & $Q^{2}$ & $Q_{b o o t}^{2}$ & $\rho$ & $F$ & $p$ \\
\hline 18 & 0.9755 & 0.9702 & 0.9613 & 0.9505 & 4.5 & 185.56 & 0.000000 \\
\hline \multicolumn{8}{|c|}{ Antifungal Activity against C. gloeosporioides: MLR Model } \\
\hline \multicolumn{8}{|c|}{$\log \left(1+\% I_{50 \mathrm{ppm}}\right)=-0.152( \pm 0.130)-0.964( \pm 0.042) \cdot S P 18+0.021( \pm 0.001) \cdot Q Y Y e-2.534( \pm 0.124) \cdot R 7 e$} \\
\hline$N$ & $R^{2}$ & $R^{2} A d j$. & $Q^{2}$ & $Q_{b o o t}^{2}$ & $\rho$ & $F$ & $p$ \\
\hline 18 & 0.9814 & 0.9774 & 0.9698 & 0.9325 & 4.5 & 245.81 & 0.000000 \\
\hline
\end{tabular}

meta-substituted ones in products of this kind. For example, the para-sustituted chlorophenyl propanols 3, 4, 15, and 16 show greater activity than the meta-sustituted ones $\mathbf{2 0}$ and $\mathbf{2 1}$ against the growth of $B$. cinerea. In the case of $C$. gloeosporioides, this fact is not clear.

Compounds 2-4 were the most active compounds against $C$. gloeosporioides with a 94, 96, and $100 \%$ inhibition rate, respectively, after five days at $150 \mu \mathrm{g} / \mathrm{mL}$. Interestingly, compound $\mathbf{4}$ displayed a $73 \%$ inhibition at the lower concentration assayed, 50 $\mu \mathrm{g} / \mathrm{mL}$.

Cyclopropyl derivatives 9-12 displayed several levels of inhibition against $C$. gloeosporioides. Surprisingly, $(S)-4^{\prime}$-chlorophenyl cyclopropyl methanol (12) exhibited weak antifungal activity, while ketone $\mathbf{9}$ and racemic alcohol $\mathbf{1 0}$ displayed high inhibition, $86 \%$ and $80 \%$, respectively, at $150 \mu \mathrm{g} / \mathrm{mL}$ after 5 days.

Moderate activity was observed for chlorophenyl propanols 14-16 and 19-21 against the growth of $C$. gloeosporioides. In both cases, with the chain in para or meta position, the $(R)$ enantiomers $\mathbf{1 6}$ and $\mathbf{2 1}$ exhibited greater inhibition of the growth of this fungus.

Compounds $\mathbf{2 - 4}$ and $\mathbf{9 - 1 1}$ were the most active compounds against $B$. cinerea, exhibiting total inhibition after 5 days at 100 $\mu \mathrm{g} / \mathrm{mL}$, and even compound 4 displayed $99 \%$ inhibition at $50 \mu \mathrm{g} /$ $\mathrm{mL}$ after 5 days.

Chlorophenyl propanols 14-16 and 19-21 displayed high antifungal activity against the growth of $B$. cinerea, especially $(R)$ (+)-1-(4'-chlorophenyl)propan-1-ol (16), showing almost complete inhibition at $150 \mu \mathrm{g} / \mathrm{mL}$ for 4 days and, interestingly, $74 \%$ inhibition at $50 \mu \mathrm{g} / \mathrm{mL}$ after 5 days.

All of the fungitoxic compounds were tested in vivo on the plant Phaseolus vulgaris, and no phytotoxic effect were observed (37).

These results seem to confirm that the presence of a hydroxyl group is fundamental for the expression of the antifungal activity of the compounds studied because it increases the potency of this class of compounds as inhibitors of the plant pathogens $B$. cinerea and C. gloeosporioides.

In order to quantitatively extend these results, a multiobjective optimization study was conducted considering the antifungal activity of this set of chlorophenyl derivatives against the two microorganisms at the same time. The MOOP-DESIRE methodology was employed for this purpose, optimizing and ranking these candidates in accordance with their global antifungal profile.

Following the strategy outlined previously $(13,14)$, we began by seeking the best linear models relating each property to the DRAGON molecular descriptors. One MLR-based PM containing 3 variables previously selected by GA was developed for both properties. The resulting best-fit models are given in Table 4 together with the statistical regression parameters. As can be observed, the models are good both in terms of their statistical significance and predictive ability (see Table 4). No violations of the basic MLR assumptions were found that could compromise the reliability of the resulting predictions (see Supporting Information for details).

Another aspect to consider in PM development is the establishment of the applicability domain of the models. Such an analysis is useful for detecting, at the same time, the presence of potential outliers and compounds with leverage value $\left(>h^{*}\right)$ from the training set that influences model parameters to a marked extent resulting in an unstable model. In this sense, it is of great importance to highlight that only predicted data for chemicals belonging to the chemical domain of the training set should be proposed. The same principle must be followed for further design of new potent antifungal compounds.

Figure 1 shows the corresponding leverage plots establishing the applicability domain inside a squared area within \pm 2 standard deviations and a leverage threshold $h^{*}$ of 0.667 (38). According to the analysis, compounds $\mathbf{1 7}$ and $\mathbf{1 3}$ were considered outliers in the models targeting their activity against $B$. cinerea and $C$. gloeosporioides, respectively, because their standardized residual values were greater than two standard deviation units. However, considering the excellent values displayed by the statistical parameters of both models, it can be inferred that these compounds do not affect the predictability of the models but rather enrich it with their structural information. In addition, a less rigorous criterion to classify a compound as an outlier has been previously established considering the threshold value for the standardized residuals equal to three standard deviation units (39), which is not reached by these chemicals. For these reasons, we decided not to eliminate them.

Because of the large amount of statistical information resulting from developing the MOOP-DESIRE methodology as well as the number of intermediate variables generated, only the essence and the possible application of the final results are going to be discussed in this section. For more details of the results from the in-between steps of the approach, the Supporting Information can be consulted.

The overall desirability function exhibits good statistical quality as indicated by $R_{D}^{2}(0.8185)$ and $A d j . R_{D}^{2}(0.8072)$. Moreover, a $Q_{D}^{2}$ value of 0.789 provides an adequate level of reliability regarding the method for predicting $D$.

The final computed values of $D_{i}, \Delta_{i}$, and the normalized values of $\Delta_{i}\left({ }^{D} \Delta_{i}\right)$ from the library of compounds used for ranking are detailed in Table 5. 


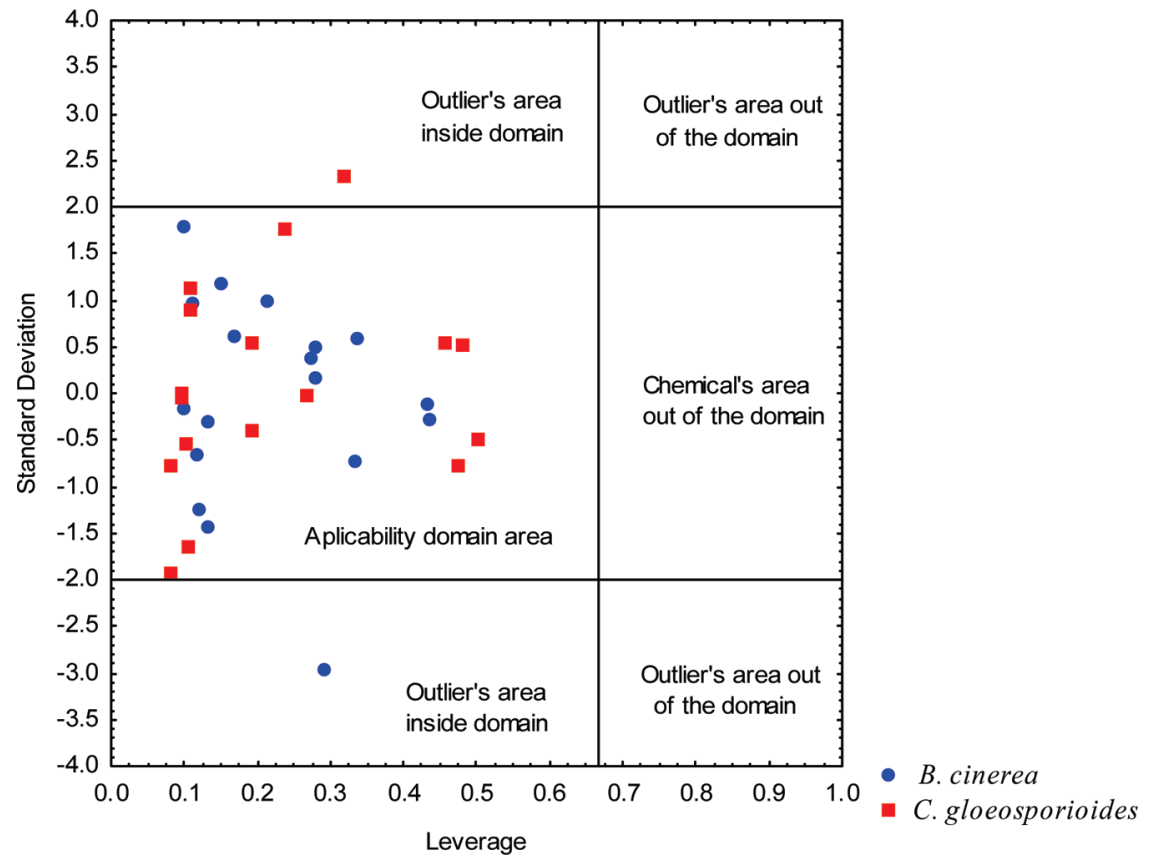

Figure 1. Applicability domain of the model.

Table 5. $\Delta_{i},{ }^{D} \Delta_{i}$, and $D_{i}$ Values of the Library of Compounds Used for Ranking

\begin{tabular}{llll}
\hline comp. & \multicolumn{1}{c}{$\Delta_{i}$} & \multicolumn{1}{c}{${ }^{D} \Delta_{i}$} & \multicolumn{1}{c}{ Pred $\mathrm{D}_{\mathrm{BC}-\mathrm{C}}$} \\
\hline $\mathbf{1}$ & 2.273197709 & 0 & 0 \\
$\mathbf{3}$ & -0.274523323 & 0.611767627 & 0.977292429 \\
$\mathbf{4}$ & -0.144862331 & 0.580632979 & 0.876804877 \\
$\mathbf{5}$ & 2.994867398 & -0.173289833 & 0 \\
$\mathbf{6}$ & 2.848688283 & -0.138188796 & 0 \\
7 & 1.082188025 & 0.285989384 & 0.159648375 \\
$\mathbf{8}$ & 1.073450485 & 0.288087472 & 0.157793888 \\
9 & -1.8913264 & 1 & 0.204689674 \\
11 & 0.514520101 & 0.422299778 & 0.479816164 \\
12 & 0.509870557 & 0.423416243 & 0.479255256 \\
13 & 2.941737312 & -0.160532053 & 0 \\
15 & 1.218223861 & 0.253323986 & 0.524521747 \\
16 & 1.257594399 & 0.243870196 & 0.527340974 \\
17 & 2.994867361 & -0.173289825 & 0 \\
18 & 2.145090354 & 0.030761583 & 0 \\
20 & 0.750696707 & 0.365588231 & 0.426903283 \\
21 & 0.752630419 & 0.365123901 & 0.435102901 \\
$\mathbf{2 2}$ & 2.928732832 & -0.157409372 & 0 \\
\hline
\end{tabular}

On the basis of $\Delta_{i}$, it is possible to arrive at a ranking of the chlorophenyl derivatives library with a corrected ranking quality index $\left(\Psi^{*}\right)$ of 0.296 representing a percentage of ranking quality $\left(R_{\%}\right)$ of $70.37(13)$. This ranking, compared with the perfect ranking, is shown in Figure 2. For more details, Table S5 and Figure S2 of the Supporting Information can be consulted.

As can be seen, some of the compounds' predicted ranks coincide with the order derived from the desirability predicted response ( $D$-based ranking) $(\mathbf{6}, \mathbf{1 1}$, and $\mathbf{2 1})$, while the rank order of chemicals $1,3,4,7,13$, and 17 shows only a marginal difference of one unit in the ranking covering the entire spectrum of possible degrees of inhibition. Moreover, it is important to stress that compounds $\mathbf{3}$ and $\mathbf{4}$ are included among the most active according to the predictions, and this is in agreement with the $D$-based ranking confirming the model's reliability for future applications. Note that the quality of the ranking attained $\left(R_{\%}=70.37\right)$ is similar to the predictability values exhibited by the MOOP processes $\left(Q_{D(\text { MIC-IC50) }}^{2}=0.789\right)$. This fact indicates that the

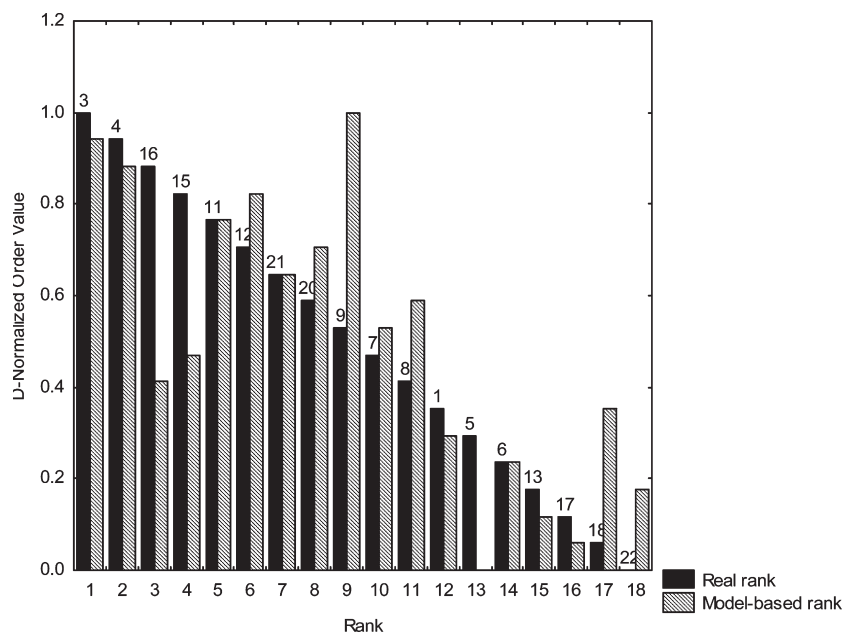

Figure 2. Comparison between the ranking from the model prediction and the one calculated from the experiment. The numbers above the bars are the identification of the molecules used in the study.

quality of both processes (desirability-based MOOP and ranking) are strongly dependent on the quality of the initial set of PMs suggesting that the ranking algorithm reflects the quality of the PMs and the MOOP process on which it is based.

It is important to note the fact that only preliminary information is obtained with in vitro bioassays, and further in vivo experiments with selected antifungal derivatives will be necessary to actually determine the disease control ability of these compounds on each pathosystem.

In summary, the activity displayed by the chlorophenyl derivatives studied here demonstrates that the presence of a hydroxyl group is fundamental for the expression of the antifungal activity of these molecules. Maximum inhibition of the growth of both phytopathogenic fungi $B$. cinerea and $C$. gloeosporioides was shown for compounds $(S)-1-\left(4^{\prime}\right.$-chlorophenyl)-2-phenylethanol (3) and (R)-1-(4'-chlorophenyl)-2-phenylethanol (4), interesting products analogous to the phytoalexin resveratrol.

As was illustrated above, the MOOP-DESIRE methodology can be used as a rational strategy to screen new fungicide 
candidates from combinatorial libraries, always considering those candidates included within the applicability domain of the PMs on which the MOOP and ranking are based. It is worth noting that the compounds included in this study did not exhibit phytotoxic effects when tested in vivo. Therefore, the use of this methodology to select compounds from a library with similar structures will most likely produce safe candidates.

Supporting Information Available: Details on the confirmation of preadopted parametric assumptions, a very important aspect in the application of linear multivariate statistical-based approaches (MLR techniques), and details related to the MOOPDESIRE methodology results. This material is available free of charge via the Internet at http://pubs.acs.org.

\section{LITERATURE CITED}

(1) Duke, S. O.; Baerson, S. R.; Dayan, F. E.; Rimando, A. M.; Schffler, B. E.; Téllez, M. R.; Wedge, D. E.; Schrader, K. K.; Akey, D. H.; Arthur, F. H.; De Lucca, A. J.; Gibson, D. M.; Harrison, H. F.Jr.; Peterson, J. K.; Gealy, D. R.; Tworkoski, T.; Wilson, C. K.; Moris, J. B. United States Department of Agriculture-Agricultural Research Service research on natural products for pest management. Pest. Manag. Sci. 2003, 59, 708-717.

(2) Echeverri, F.; Torres, F.; Quiñones, W.; Cardona, G.; Archbold, R.; Roldan, J.; Brito, I.; Luis, J. G.; Lahlou, E. H. Danielone, a phytoalexin from papaya fruit. Phytochemistry 1997, 44, 255-256.

(3) Bustillo, A. J.; Aleu, J.; Hernández-Galán, R.; Collado, I. G. Biocatalytically assisted preparation of antifungal chlorophenylpropanols. Tetrahedron: Asymmetry 2002, 13, 1681-1686.

(4) Coley-Smith, J. R.; Verhoeff, K.; Jarvis, W. R. The Biology of Botrytis; Academic Press: London, 1980.

(5) Sutton, B. C. In Colletotrichum, Biology, Pathology and Control; CAB International: Oxford, England, 1992; pp 1-26.

(6) Aleu, J.; Hernández-Galán, R.; Hanson, J. R.; Hitchcock, P. B.; Collado, I. G. Biotransformation of the fungistatic sesquiterpenoid ginsenol by Botrytis cinerea. J. Chem. Soc., Perkin Trans. 1 1999, 727-730.

(7) Bustillo, A. J.; Aleu, J.; Hernández-Galán, R.; Collado, I. G. Biotransformation of the fungistatic compound $(R)-(+)-1-\left(4^{\prime}\right.$-chlorophenyl)propan-1-ol by Botrytis cinerea. J. Mol. Catal. B: Enzym. 2003, 21, 267-271.

(8) Aleu, J.; Hanson, J. R.; Galán, R. H.; Collado, I. G. Biotransformation of the fungistatic sesquiterpenoid patchoulol by Botrytis cinerea. J. Nat. Prod. 1999, 62, 437-40.

(9) Aleu, J.; Hernández-Galán, R.; Collado, I. G. Biotransformation of the fungistatic sesquiterpenoid isoprobotryan- $9 \alpha-$ ol by Botrytis cinerea. J. Mol. Catal B: Enzym. 2002, 16, 249-253.

(10) Bustillo, A. J.; García-Pajón, C. M.; Aleu, J.; Hernández-Galán, R.; Collado, I. G. Studies on biotransformation of $( \pm)-1-\left(4^{\prime}\right.$-chlorophenyl)2-phenylethanol. Tetrahedron: Asymmetry 2003, 14, 3755-3760.

(11) Saiz-Urra, L.; González, M. P.; Collado, I. G.; Hernández-Galán, R. Quantitative structure-activity relationship studies for the prediction of antifungal activity of $\mathrm{N}$-arylbenzenesulfonamides against Botrytis cinerea. J. Mol. Graphics Modell. 2007, 25, 680-690.

(12) Reino, J. L.; Saiz-Urra, L.; Hernández-Galán, R.; Aran, V. J.; Hitchcock, P. B.; Hanson, J. R.; Pérez González, M.; Collado, I. G. Quantitative structure: Antifungal activity relationships of some benzohydrazides against Botrytis cinerea. J. Agric. Food Chem. 2007, 55, 5171-5179.

(13) Cruz-Monteagudo, M.; Borges, F.; Cordeiro, M. N.; Cagide Fajin, J. L.; Morell, C.; Ruiz, R. M.; Canizares-Carmenate, Y.; Domínguez, E. R. Desirability-based methods of multiobjective optimization and ranking for global QSAR studies. Filtering safe and potent drug candidates from combinatorial libraries. J. Comb. Chem. 2008, 10, 897-913.

(14) Cruz-Monteagudo, M.; Borges, F.; Cordeiro, M. N. Desirabilitybased multiobjective optimization for global QSAR studies: application to the design of novel NSAIDs with improved analgesic, antiinflammatory and ulcerogenic profiles. J. Comput. Chem. 2008, $29,2445-59$.

(15) De Boor, C. A Practical Guide to Splines; Springer-Verlag: New York, 1978

(16) Gerald, C. F.; Wheatley, P. O. Applied Numerical Analysis, 4th ed.; Addison Wesley: Reading, MA, 1989.

(17) STATISTICA, 6.0 for Windows; Statsoft_Inc.: Tulsa, OK, 2001.

(18) Coleman, T. F.; Li, Y. An interior, trust region approach for nonlinear minimization subject to bounds. SIAM J. Optim. 1996, $6,418-445$.

(19) Coleman, T. F.; Li, Y. On the convergence of reflective Newton methods for large-scale nonlinear minimization subject to bounds. Math. Program 1994, 67, 189-224.

(20) MATLAB, 7.2; The MathWorks, Inc.: Natick, MA, 2006.

(21) Burkert, U.; Allinger, N. L. Molecular Mechanics; American Chemical Society: Washington, DC, 1982.

(22) Clark, T. Computational Chemistry; Wiley: New York, 1985.

(23) ChemDraw Ultra, 9.0; CambridgeSoft: Cambridge, MA, 2004.

(24) Frank, J. MOPAC, 6.0; Seiler Research Laboratory, US Air Force Academy: Colorado Springs, CO, 1993.

(25) Todeschini, R.; Consonni, V.; Pavan, M. DRAGON Software, 2.1; Milano Chemometrics: Milano, Italy, 2002.

(26) Leardi, R.; Boggia, R.; Terrile, M. Genetic algorithms as a strategy for feature selection. J. Chemom. 1992, 6, 267-281.

(27) Yasri, A.; Hartsough, D. Toward an optimal procedure for variable selection and QSAR model building. J. Chem. Inf. Comput. Sci. 2001, $41,1218-27$.

(28) Hou, T. J.; Wang, J. M.; Liao, N.; Xu, X. J. Applications of genetic algorithms on the structure-activity relationship analysis of some cinnamamides. J. Chem. Inf. Comput. Sci. 1999, 39, 775-81.

(29) Hasegawa, K.; Kimura, T.; Funatsu, K. GA strategy for variable selection in QSAR studies: application of GA-based region selection to a 3D-QSAR study of acetylcholinesterase inhibitors. J. Chem. Inf. Comput. Sci. 1999, 39, 112-20.

(30) Barbosa de Oliveira, D.; Gaudio, A. C. BuildQSAR; Physics Department-CCE, University of Espírito Santo: Vitória ES, Brasil, 2000.

(31) Barbosa, D.; Gaudio, A. C. BuildQSAR: A new computer program for QSAR analysis. Quant. Struct.-Act. Relat. 2000, 19, 599-601.

(32) Cruciani, G.; Baroni, M.; Clementi, S.; Costantino, G.; Riganelli, D.; Skagerberg, B. Predictive ability of regression models. Part I: Standard deviation of prediction errors (SDEP). J. Chemom. 1992, 6, 335-346.

(33) Van Waterbeemd, H. Chemometric Methods in Molecular Design; Wiley-VCH: New York, 1995; Vol. 2.

(34) Efron, B. Better Bootstrap Confidence Intervals. J. Am. Stat. Assoc. 1987, $82,171-200$

(35) Collado, I. G.; Hanson, J. R.; Macías-Sánchez, A. J. Spanish Patent ES 2154185, 2001; Spanish Pat. Appl. 1998-2241, 1998; Chem. Abstr. 2001, 135, 195688.

(36) Patil, I. S.; Kulkarni, S.; Hedge, R. K. Bioassay of fungicides against Drechslera sorokiniana (Sacc). Pesticides 1986, 20, 30-31.

(37) Colmenares, A. J.; Aleu, J.; Durán Patrón, R.; Collado, I. G.; Hernández-Galán, R. The putative role of botrydial and related metabolites in the infection mechanism of Botrytis cinerea. J. Chem. Ecol. 2002, 28, 997-1005.

(38) Eriksson, L.; Jaworska, J.; Worth, A. P.; Cronin, M. T.; McDowell, R. M.; Gramatica, P. Methods for reliability and uncertainty assessment and for applicability evaluations of classification- and regression-based QSARs. Environ. Health Perspect. 2003, 111, 13611375.

(39) Gramatica, P. Principles of QSAR models validation: internal and external. QSAR Comb. Sci. 2007, 26, 694-701.

Received February 3, 2009. Revised manuscript received April 27, 2009. Financial support from the Spanish Science and Technology Ministry through project AGL2006-13401-C02-01 and the Junta de Andalucía through project PAI05-FQM-00489 is gratefully acknowledged. 